\title{
NOUVELLE
}

\section{Relation entre durée du sommeil chez l'adulte et risque de démence}

\author{
Séverine Sabia ${ }^{1,2}$, Julien Dumurgier ${ }^{1,3}$
}

\author{
lépidémiologie du vieillissement et des maladies \\ neurodégénératives, Université de Paris, Inserm U1153, \\ 10 avenue de Verdun, 75010 Paris, France. \\ 2Department of epidemiology and public health, \\ University college London, Londres, Royaume-Uni. \\ ${ }^{3}$ Centre de neurologie cognitive, hôpital Lariboisière - \\ Fernand-Widal, Assistance publique-Hôpitaux de Paris, \\ Université de Paris, Paris, France. \\ severine.sabia@inserm.fr
}

> La démence, dont la maladie d'Alzheimer est la principale cause [1], touchait environ 1,2 million de personnes en France en $2014^{1}$. Avec l'augmentation de l'espérance de vie, ce nombre pourrait doubler d'ici à 2050, faisant de la démence un enjeu majeur de santé publique [2]. Il est estimé que 50 à $90 \%$ des personnes atteintes de démence ont des troubles du sommeil. Ces troubles sont différents de ceux rencontrés au cours du vieillissement normal, et se caractérisent par un endormissement plus tardif, de longues périodes d'éveil durant la nuit et plusieurs phases de sommeil durant la journée, dénotant une nette désynchronisation de l'horloge interne avec le cycle jour/nuit [3]. La dégradation majeure du sommeil nocturne contribue d'ailleurs fortement à la

1 https://www.santepubliquefrance.fr/maladies-ettraumatismes/maladies-neurodegeneratives/maladie- $d$ alzheimer-et-autres-demences/la-maladie/\#tabs décision de placement institutionnel des patients atteints de démence. Certaines études suggèrent également l'existence de changements dans la qualité et la quantité de sommeil dans les années qui précèdent le diagnostic de la démence. II est à noter que des altérations cérébrales et des processus neurodégénératifs liés à la démence peuvent apparaître jusqu'à 20 ans avant sa manifestation clinique [4]. Ainsi, il est difficile de savoir si les troubles du sommeil en phase préclinique de la démence sont une manifestation de la maladie, ont des causes communes avec celles de la démence, ou bien s'ils jouent un rôle dans sa progression.

Une association bidirectionnelle entre sommeil et maladie d'Alzheimer a été proposée [5]. Des études expérimentales chez l'homme et la souris ont montré qu'une durée de sommeil réduite, ainsi qu'un sommeil fragmenté seraient associés à une augmentation de l'activité neuronale conduisant à une accu- mulation du peptide $\beta$-amyloïde et une altération du système glymphatique en charge du drainage des déchets cérébraux, dont le peptide $\beta$-amyloïde et la protéine tau impliqués dans le développement de la maladie d'Alzheimer. Une détérioration du rythme circadien est également observée en parallèle de la progression de la maladie d'Alzheimer en phase préclinique [6]. II reste à savoir si des interventions visant à améliorer le sommeil nocturne pourraient retarder les manifestations cliniques de la démence.

L'hypothèse d'une association entre sommeil et démence suggère que le sommeil, de nombreuses années avant la survenue de la démence, et donc avant sa phase préclinique, pourrait également être impliqué dans le développement de la démence. Peu d'études ont examiné l'association entre la durée de sommeil en milieu de vie et la survenue de la démence au cours 


\begin{tabular}{|c|c|c|}
\hline & $\begin{array}{l}\text { Nombre de cas / } \\
\text { Nombre total }\end{array}$ & $\begin{array}{l}\text { Hazard ratio } \\
\text { (intervalle de confiance à } 95 \% \text { ) }\end{array}$ \\
\hline \multicolumn{3}{|c|}{ Durée de sommeil à $\mathbf{5 0}$ ans (suivi moyen = 25 ans) } \\
\hline Courte $: \leqslant 6$ heures & $211 / 3149$ & $1,22(1,01-1,48)$ \\
\hline Normale : 7 heures & $219 / 3624$ & $l$ (ref) \\
\hline Longue : $\geqslant 8$ heures & $91 / 1186$ & $1,25(0,98-1,60)$ \\
\hline \multicolumn{3}{|c|}{ Durée de sommeil à 60 ans (suivi moyen = 15 ans) } \\
\hline Courte : $\leqslant 6$ heures & $192 / 2759$ & $1,37(1,10-1,72)$ \\
\hline Normale : 7 heures & $142 / 2988$ & 1 (ref) \\
\hline Longue : $\geqslant 8$ heures & $75 / 1417$ & $1,15(0,87-1,52)$ \\
\hline \multicolumn{3}{|c|}{ Durée de sommeil à 70 ans (suivi moyen = 8 ans) } \\
\hline Courte $: \leqslant 6$ heures & $171 / 2429$ & $1,24(0,98-1,57)$ \\
\hline Normale : 7 heures & $131 / 2578$ & $l(\mathrm{ref})$ \\
\hline Longue : $\geqslant 8$ heures & $90 / 1509$ & $1,15(0,88-1,51)$ \\
\hline \multicolumn{3}{|c|}{ Trajectoires de durée de sommeil entre 50 et 70 ans (suivi moyen = 7 ans) } \\
\hline Durée courte de façon persistante & $103 / 1358$ & $1,30(1,00-1,69)$ \\
\hline Durée normale de façon persistante & $141 / 2520$ & $l($ ref) \\
\hline Durée longue de façon persistante & $35 / 461$ & $1,28(0,88-1,85)$ \\
\hline Changement de durée courte à normale & $61 / 1086$ & $1,20(0,89-1,63)$ \\
\hline Changement de durée normale à longue & $47 / 946$ & $1,02(0,73-1,42)$ \\
\hline Changement de durée normale à courte & $39 / 504$ & $1,13(0,79-1,62)$ \\
\hline \multicolumn{3}{|c|}{ Durée de sommeil, mesurée par accéléromètre en 2012 ou en 2013 (suivi moyen = 6 ans) } \\
\hline Tertile $1:<6$ heures 14 minutes & $53 / 1296$ & $1,63(1,04-2,57)$ \\
\hline Tertile $2: 6$ heures 14 minutes à 7 heures & $31 / 1296$ & $l($ ref) \\
\hline Tertile $3: \geqslant 7$ heures & $27 / 1296$ & $0,78(0,46-1,32)$ \\
\hline
\end{tabular}

Tableau I. Association entre durée de sommeil et risque de démence. Hazard ratio : rapport de risques instantanés (souvent assimilé au risque relatif) de démence par rapport au groupe de référence (ref), estimé à partir de modèles de Cox (modèle à risque proportionnel) ajusté sur les facteurs sociodémographiques (âge, sexe, ethnicité, éducation, statut matrimonial), sur les comportements liés au mode de vie (tabagisme, consommation d’alcool, activité physique, consommation de fruits et légumes), sur les facteurs de risque cardio-métaboliques (indice de masse corporelle, hypertension artérielle, diabète), sur les maladies cardiovasculaires, et sur la santé mentale (échelle de dépression et médicaments du système nerveux central).

$\mathrm{du}$ vieillissement, et leurs résultats ne permettaient pas de conclure, en partie en raison du petit nombre de personnes enrôlées dans les catégories «durée de sommeil courte» et «durée de sommeil longue ». Nous avons donc étudié l'association entre la durée du sommeil à différents âges de la vie adulte et le risque de survenue de démence [7].
L'étude portait sur 7959 participants de la cohorte britannique Whitehall II study, suivis depuis leur inclusion dans la cohorte en 1985 jusqu'en $2019^{2}$. Ces per-

${ }^{2}$ La cohorte Whitehall II a été mise en place en 1985. II s'agit d'une enquête de cohorte prospective menée auprès de 10308 fonctionnaires britanniques (6895 hommes et 3413 femmes) basés à Londres et âgés de 34 à 56 ans lors de leur inclusion dans la cohorte. sonnes ont répondu à un questionnaire sur la durée habituelle du sommeil à six reprises au cours du suivi. Ces données ont permis d'extraire la durée de sommeil aux âges spécifiques de 50, 60, et 70 ans, que nous avons catégorisé en: $\leqslant 6$ heures, 7 heures, et $\geqslant 8$ heures par nuit. Par ailleurs, un lien avec les bases électroniques de santé au Royaume-Uni a 
permis d'obtenir le statut de démence et la date de son diagnostic, le cas échéant, pour chaque participant jusqu'en mars 2019. À partir de régressions de $\mathrm{Cox}^{3}$ avec l'âge en échelle de temps, nous avons ainsi pu étudier l'association entre la durée de sommeil à 50, 60, 70 ans, ainsi que l'évolution de la durée de sommeil entre ces âges, et la survenue de la démence. Les modèles de régression prenaient en compte également le sexe, le niveau d'étude, le statut matrimonial, les facteurs de risque cardio-métaboliques, la prévalence de maladies cardiovasculaires, la prise de médicaments agissant sur le système nerveux central, et les symptômes dépressifs au moment de la mesure de la durée de sommeil.

Parmi les 7959 participants inclus dans l'étude, 521 ont développé une démence sur une période moyenne de suivi de 25 ans (écart-type $=7$ ans). Le taux d'incidence pour 1000 personnes-années était le plus bas pour les personnes qui rapportaient dormir 7 heures par nuit, que ce soit à l'âge de 50 , de 60 , ou de 70 ans. Les résultats ont montré un risque de démence augmenté de $22 \%$ (intervalle de confiance : 1 à $48 \%$ ) et $37 \%$ (intervalle de confiance : 10 à $72 \%$ ) chez les personnes qui dormaient 6 heures ou moins par nuit, respectivement, à 50 et 60 ans, par rapport aux personnes dormant 7 heures par nuit aux mêmes âges. Lorsque la trajectoire de durée de sommeil entre 50 et 70 ans était considérée, un risque de démence augmenté de $30 \%$ (intervalle de confiance : 0 à $69 \%$ ) était observé chez les individus dormant 6 heures ou moins de façon persistante dans cette tranche d'âge par rapport à ceux dormant 7 heures par nuit sur la même période de la vie. Parmi les personnes dormant 8 heures ou plus de façon régulière, une augmentation du risque était également suggérée, mais l'association n'atteignait pas le seuil de signification statistique. Afin de tester la robustesse des résultats, plusieurs analyses supplémentaires ont été faites.

\footnotetext{
3 Ces modèles évaluent le temps écoulé avant qu'u
} événement ne survienne en fonction de différents critères.
L'association entre une durée de sommeil courte et le risque de démence se retrouvait également chez les personnes n'ayant pas d'antécédent de maladie mentale (dépression, troubles de l'humeur ou du comportement) ou bien lorsqu'une mesure objective de la durée de sommeil par accéléromètre était utilisée (analyse restreinte à un sous-groupe de 3888 participants ayant porté un accéléromètre pendant 9 jours) (Tableau I).

On connaissait déjà l'impact à courtterme du manque de sommeil sur la mémoire et le raisonnement les lendemains de nuits courtes. Cette étude montre que le manque de sommeil de façon habituelle et persistante dans la période du milieu de la vie pourrait avoir un impact sur le risque de démence à un âge plus avancé. La promotion d'une «bonne nuit de sommeil » pourrait être bénéfique pour la prévention de la démence. Une bonne hygiène du sommeil passe par une bonne hygiène de vie en général, comme la pratique régulière d'une activité physique et un régime alimentaire sain, la mise en place d'un rythme régulier d'heure du coucher, et l'éviction d'exposition aux écrans et lumières fortes dans la demi-heure qui précède l'heure du coucher. Ces habitudes de vie favorisent un meilleur endormissement et une bonne qualité du sommeil.

Il est important de placer ces résultats dans le contexte plus général de la prévention de la démence. En effet, il s'agit d'une maladie multifactorielle, qui résulte probablement de l'accumulation de plusieurs facteurs de risque tout au long de la vie plutôt que d'un seul facteur pris isolément. Ces facteurs de risque restent encore en partie à identifier. II a été suggéré que le maintien d'habitudes de vie saines sans tabac ni forte consommation d'alcool, d'une bonne santé cardiovasculaire sans facteurs de risque, tels qu'obésité, hypertension artérielle, ou diabète, et de contacts sociaux dès l'âge de 50 ans permettrait de réduire le risque de démence $[8,9](\rightarrow)$.

$(\rightarrow)$ Voir la Nouvelle de S. Sabia et A. Singh-Manoux, $m / s$ $n^{\circ} 2$, février 2020, page 101
La durée du sommeil vient donc s'ajouter à la liste des facteurs pouvant influencer le risque de démence. Reste à savoir si ces facteurs jouent un rôle indépendant les uns des autres ou s'ils agissent de façon synergique, et quels sont les mécanismes qui sous-tendent leur association avec la survenue de la démence, afin de pouvoir envisager des stratégies de prévention ciblées. $\diamond$ Association between sleep duration in adulthood and risk of dementia

\section{LIENS D’INTÉRÊT}

Les auteurs déclarent n'avoir aucun lien d'intérêt concernant les données publiées dans cet article.

\section{RÉFÉRENCES}

1. Dumurgier J, Sabia S. Nouvelles tendances épidémiologiques de la maladie d'Alzheimer. Rev Prat $2020 ; 70: 149-51$.

2. Mura T, Dartigues JF, Berr C. How many dementia cases in France and Europe? Alternative projections and scenarios 2010-2050. Eur J Neurol 2010 ; 17 : 252-9.

3. Duncan MJ. Interacting influences of aging and Alzheimer's disease on circadian rhythms. Eur J Neurosci $2020 ; 51: 310-25$.

4. Jack CR Jr, Knopman DS, Jagust WJ, et al. Tracking pathophysiological processes in Alzheimer's disease: an updated hypothetical model of dynamic biomarkers. Lancet Neurol 2013 ; 12 : 207-16.

5. Ju YE, Lucey BP, Holtzman BM. Sleep and Alzheimer disease pathology: a bidirectional relationship. Nat Rev Neurol 2014 ; 10 : 115-9.

6. Li P, Gao L, Gaba A, et al. Circadian disturbances in Alzheimer's disease progression: a prospective observational cohort study of community-based older adults. Lancet Healthy Longev $2020 ; 1$ : ع96-105.

7. Sabia S, Fayosse A, Dumurgier J et al. Association of sleep duration in middle and old age with incidence of dementia. Nat Commun $2021 ; 12: 2289$.

8. Sabia S, Fayosse A, Dumurgier J et al. Association of ideal cardiovascular health at age 50 with incidence of dementia: 25 year follow-up of Whitehall II cohort study. BMJ $2019 ; 366$ : 14414.

9. Sabia S, Singh-Manoux A. Association entre la fréquence des contacts sociaux durant la vie adulte et le risque de démence au cours du vieillissement. Med Sci (Paris) 2020 ; 36 : 101-3.
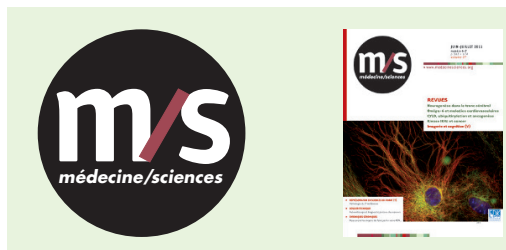

Abonnez-vous

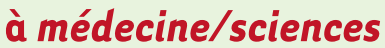

Bulletin d'abonnement page 1194 dans ce numéro de $\mathrm{m} / \mathrm{s}$ 